\title{
Temperature Evolution of Optical Behaviors of $\beta$-HMX Single Crystals from Spectroscopy \\ Ellipsometry
}

\author{
Pangangjing Zou ${ }^{1}$, Xiangqi Wang ${ }^{2 *}$, Lin Zhang ${ }^{1,3}$, Rucheng Dai ${ }^{4}$, Zhongping Wang ${ }^{4}$, \\ Yu Liu ${ }^{1}$, Zengming Zhang ${ }^{4,5}$, Shiliang Huang ${ }^{1 *}$, and Jun Tan ${ }^{2 *}$ \\ ${ }^{1}$ Institute of Chemical Materials, China Academy of Engineering Physics, Mianyang \\ 621900, China \\ ${ }^{2}$ Jihua Laboratory Testing Center, Jihua Laboratory, Foshan 528000, China \\ ${ }^{3}$ School of Chemical Engineering and Technology, Tianjin University, Tianjin \\ 300072, China \\ ${ }^{4}$ The Centre for Physical Experiments, University of Science and Technology of \\ China, Hefei 230026, China \\ ${ }^{5}$ Department of Physics, University of Science and Technology of China, Hefei \\ 230026, China \\ *Corresponding Authors: wangxq@jihualab.com; shilianghuang@caep.cn; \\ tanjun@jihualab.com
}

\section{List of Contents:}

Figure S1 The molecular arrangements on the (a) (110) and (b) (011) planes of $\beta$-HMX.

Figure S2 Statistics of surface roughness for $\beta$-HMX with (110) and (011) orientations, respectively. 


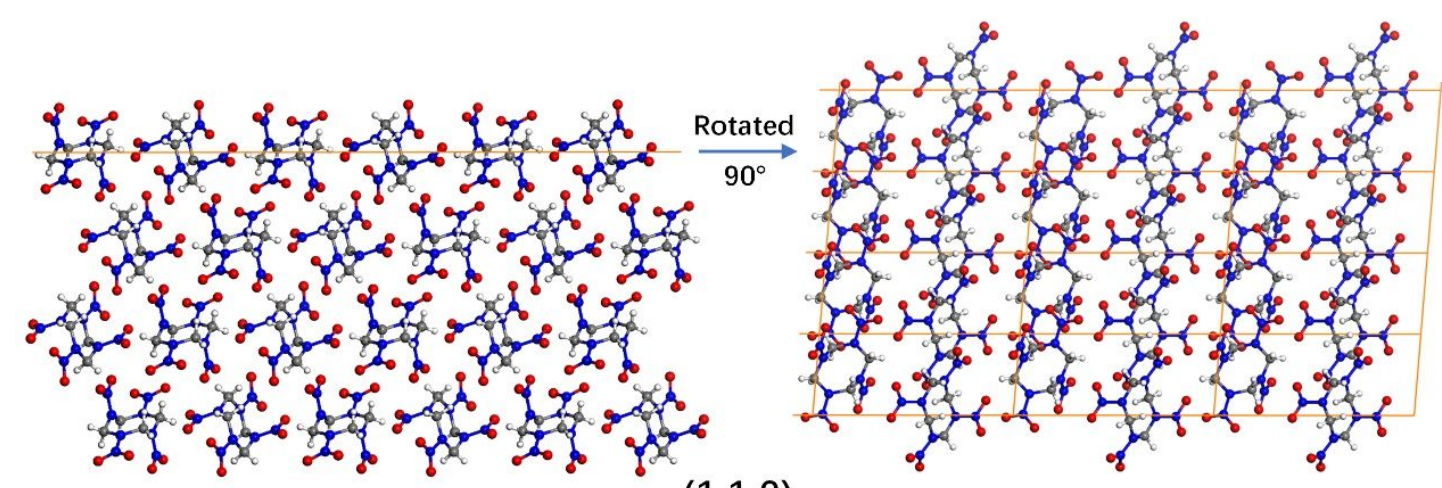

(1 110$)$

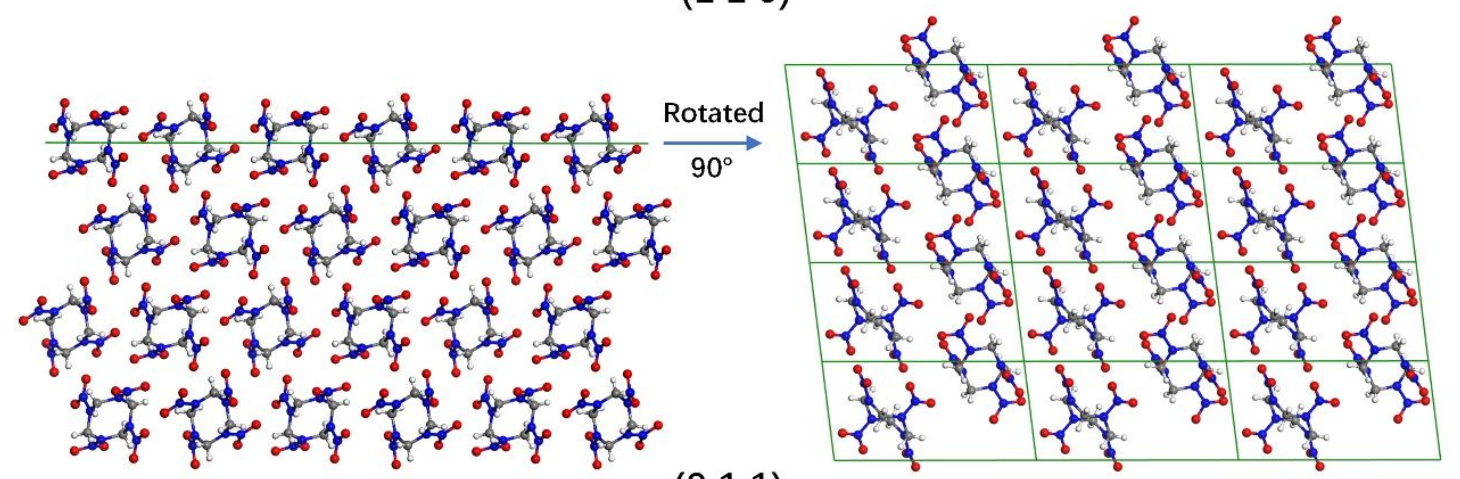

(0 11 1)

Figure S1 The molecular arrangements on the (a) (110) and (b) (011) planes of $\beta$-HMX cleaved from the crystal structure of $\beta$-HMX in space group $P 2_{1} / n$ with $a=6.54 \AA, b=11.05 \AA, c=7.73 \AA$ and $\beta=102.8^{\circ}$.

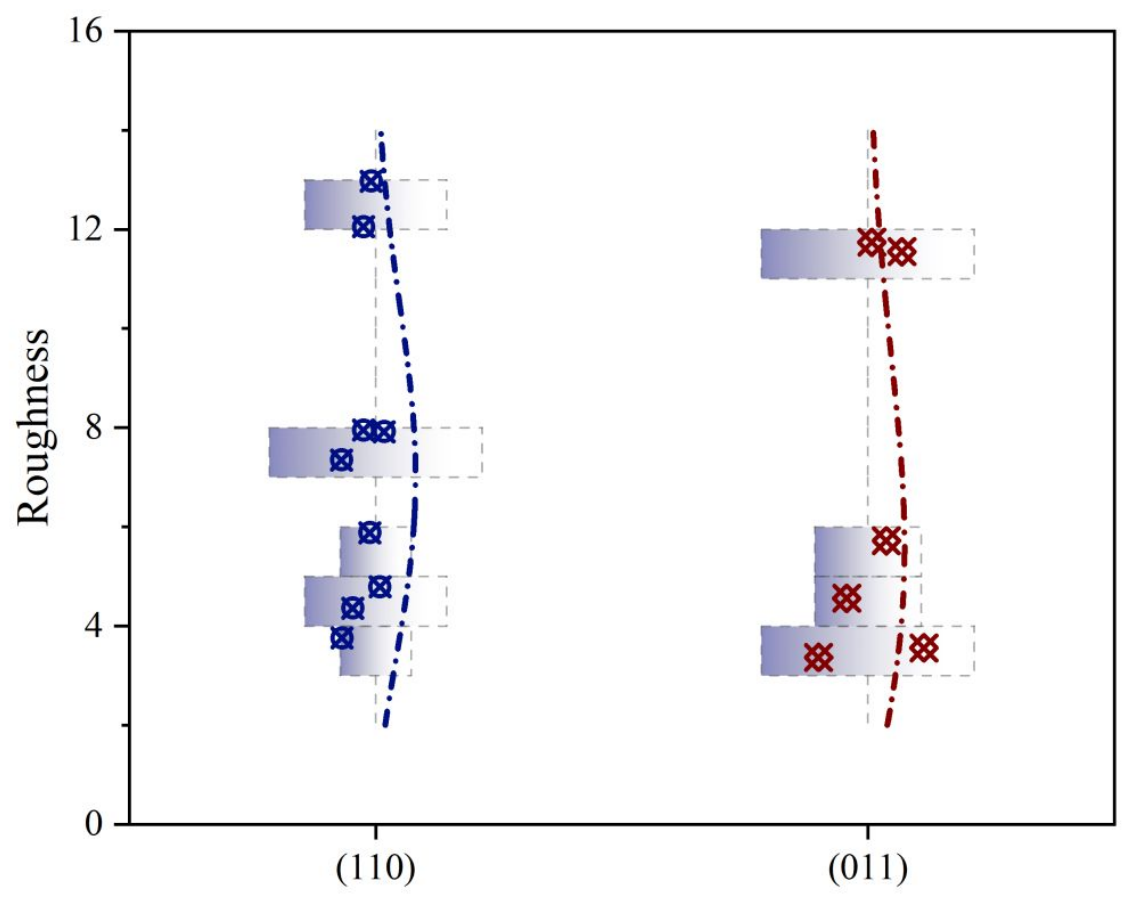

Figure S2 Statistics of surface roughness for $\beta$-HMX with (110) and (011) 
orientations, respectively. 